\title{
Real-time detection, classification, and quantification of apneic episodes using miniature surface motion sensors in rats
}

\author{
Dan Waisman 1,2, Lior Lev-Tov ${ }^{2,3}$, Carmit Levy ${ }^{3-5}$, Anna Faingersh ${ }^{3}$, Ifat Colman Klotzman ${ }^{4,5}$, Haim Bibi ${ }^{6}$, \\ Avi Rotschild ${ }^{1,2}$ and Amir Landesberg ${ }^{3}$
}

BACKGROUND: Real-time detection and classification of apneic episodes remain significant challenges. This study explores the applicability of a novel method of monitoring the respiratory effort and dynamics for rapid detection and classification of apneic episodes.

METHODS: Obstructive apnea (OA) and hypopnea/central apnea (CA) were induced in nine tracheostomized rats, by short-lived airway obstruction and administration of succinylcholine, respectively. Esophageal pressure (EP), EtCO, arterial $\mathrm{O}_{2}$ saturation $\left(\mathrm{SpO}_{2}\right)$, heart rate, and blood pressure were monitored. Respiratory dynamics were monitored utilizing three miniature motion sensors placed on the chest and epigastrium. Three indices were derived from these sensors: amplitude of the tidal chest wall displacement (TDi), breath time length (BTL), that included inspiration and rapid expiration phases, and amplitude time integral (ATI), the integral of breath amplitude over time.

RESULTS: OA induced a progressive $6.42 \pm 3.48$-fold increase in EP from baseline, which paralleled a 3.04 \pm 1.19 -fold increase in TDi $(P<0.0012)$, a $1.39 \pm 0.22$-fold increase in BTL $(P<0.0002)$, and a $3.32 \pm 1.40$-fold rise in the ATI $(P<0.024)$. During central hypopneic/apneic episodes, each sensor revealed a gradual decrease in TDi, which culminated in absence of breathing attempts.

CONCLUSION: Noninvasive monitoring of chest wall dynamics enables detection and classification of central and obstructive apneic episodes, which tightly correlates with the EP.

A pneic episodes frequently occur in newborn infants, especially in premature infants, and are classified as central (CA), obstructive (OA), or mixed apneic events $(1-3)$. These episodes can potentially lead to significant multisystem morbidity, such as poor neurodevelopment, retinopathy of prematurity, and impaired growth (3-5). In older infants, apneic episodes are associated with apparent life-threatening events $(6,7)$. Interestingly, the incidence rate of sudden infant death syndrome declined from 142.9 to 54.6 deaths per 100,000 live births between 1984 and 2004 (8). However, the rate of accidental suffocation and strangulation in bed, many of which are potentially preventable, increased from 2.8 to 12.5 deaths per 100,000 live births within the same time period (8). Similar trends were also reported by others (9).

Current monitoring methods in use for premature infants and sick term infants in intensive care units (ICUs) rely on measures of oral/nasal airflow, end-tidal $\mathrm{CO}_{2}\left(\mathrm{EtCO}_{2}\right)$, cardiorespiratory functions (impedance, heart rate, blood pressure), and pulse oximetry. CA diagnosis is known to be fast and effective when using these monitoring methods. However, hypopnea and OA are usually suspected only after significant hypoxemia and bradycardia are present $(4,10,11)$, as observed in infants monitored during sudden death and apparent lifethreatening events (12-14).

To date, there is no simple and precise modality for early detection of OA (15-17). While measurement of the esophageal pressure (EP) is the gold standard for quantification of the respiratory effort and for classification of apneic episodes $(18,19)$, it is invasive and causes discomfort. Polysomnography is currently the common method for evaluating the type, source, and severity of apneic episodes in sleep studies in infants and older children $(20,21)$. This technique monitors respiratory parameters, such as nasal airflow, respiratory effort (with bands around the chest and abdomen), peripheral pulse oximetry, heart rhythm (ECG), electroencephalography, eye movements, and muscle activity (electromyography). However, it is still difficult to perform polysomnography in young pediatric patients, due to lack of cooperation and technical difficulties that multiparameter recordings present (22). In addition, it is important to note that the interpretation and diagnosis of the sleep data are usually done off-line, at the end of the recording.

Recently, we have described the feasibility and clinical relevance of monitoring chest wall dynamics for early detection 
of deteriorating ventilation $(23,24)$, by means of miniature motion sensors (accelerometers) attached to both sides of the chest and abdomen of animals (23) or of newborn infants (24). Deteriorating ventilation was detected by monitoring the amplitude (tidal volume) and symmetry (distribution) of the ventilation $(23,24)$. The objective of the present study was to explore the utility of these miniature motion sensors in realtime monitoring, detection, and classification of central and obstructive apneic and hypopneic episodes, in spontaneously breathing rats.

\section{RESULTS}

Altogether, 43 OA events and 14 central hypopneic/apneic events were induced and monitored in nine rats (mean weight: $313 \pm 46 \mathrm{~g}$ ). However, all the events induced and monitored in each rat were considered a single experiment $(n=9)$.

Figure 1 presents the raw data from one OA experiment. OA was imposed after $153 \mathrm{~s}$ of baseline recording and was released after $24 \mathrm{~s}$. During the occlusion, no $\mathrm{EtCO}_{2}$ signals were detected, apart from a short-lived signal imposed when the sampled air shifted from the collecting tube to the sensor within the air gas monitor. The motion sensor signals corresponded well to the EP deflections during baseline, obstruction, and recovery periods. Typical changes during OA included a significant, progressive increase in the amplitude, and widening of the breath cycle, as demonstrated by both the EP and chest wall dynamics measurements.

Figure 2 presents the raw data from one central hypopneic/ apneic episode. IV succinylcholine injection began at $t=58 \mathrm{~s}$ (first arrow) and induced a progressive decrease in the respiratory effort, resulting in hypopnea at $t=113 \mathrm{~s}$ (second arrow), followed by apnea with no breath signals, at $t=208 \mathrm{~s}$ (third arrow).

\section{Tidal Displacement Index}

Figure 3 compiles the tidal displacement index (TDi) and EP measurements from the obstructive (Figure 3a,b) and CA (Figure 3c,d) experiments $(n=9)$. An overt 3.04 \pm 1.19 -fold increase $(P<0.0012)$ in TDi relative to baseline was obtained in the first breath during $\mathrm{OA}$, which further escalated during successive breath attempts, as shown in Figure 3a. In parallel, the EP amplitude significantly increased by $6.42 \pm 3.48$-fold $(P<0.0016)$ in the first breath taken during the obstruction episodes and continued to grow during successive breath attempts, as shown in Figure $3 \mathbf{b}$. As expected, an overt decrease in TDi was measured during the central apneic episodes (Figure 3c), and a similar decline in the EP was observed (Figure 3d). The duration of hypopnea that preceded apnea varied and lasted for a mean $97 \pm 99 \mathrm{~s}$ (median: $60 \mathrm{~s}$; range: 19-350 s). EP changes during both OA, and central hypopneic/apneic episodes were congruent with those observed for the TDi. The mean correlation coefficient of TDi and EP was $0.965 \pm 0.027$ (range: 0.905-0.996) for all obstructive episodes, and $0.970 \pm 0.027$ (range: $0.909-0.996$ ) for all hypopneas during pseudo-CAs. The relationships between the TDi and the EP during both obstructive and hypopneic/apneic events are presented in the Supplementary Figures S1 and S2 online, respectively.

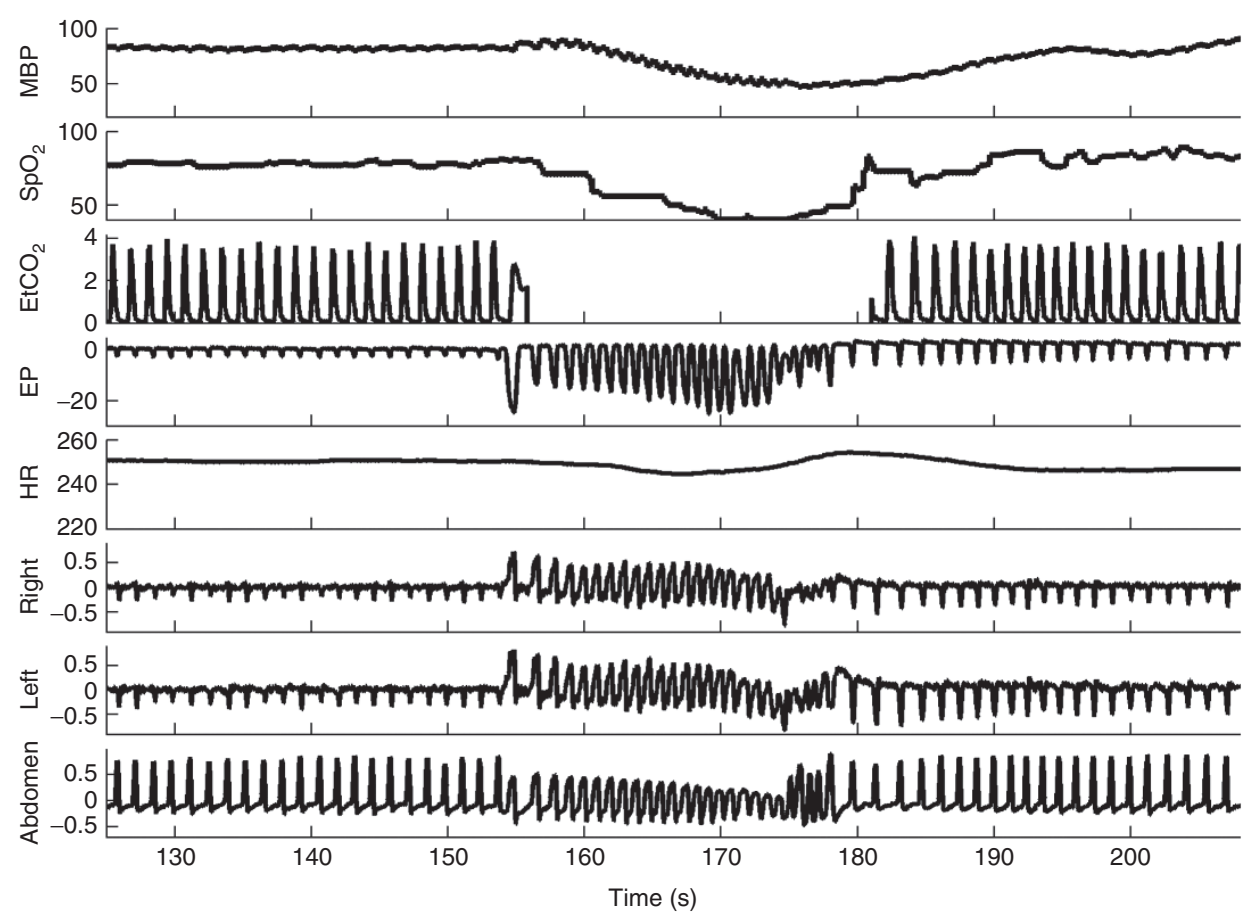

Figure 1. Airway obstruction induced a significant increase in the respiratory effort. Note the concurrent changes in esophageal pressure (EP) and in the tidal displacement indices (TDi) measured by the right, left, and abdominal sensors. $\mathrm{EtCO}_{2^{\prime}}$ end tidal $\mathrm{CO}_{2^{\prime}} \mathrm{HR}$, heart rate; $\mathrm{MBP}$, mean arterial blood pressure; $\mathrm{SpO}_{2}$, pulse oximetry. 


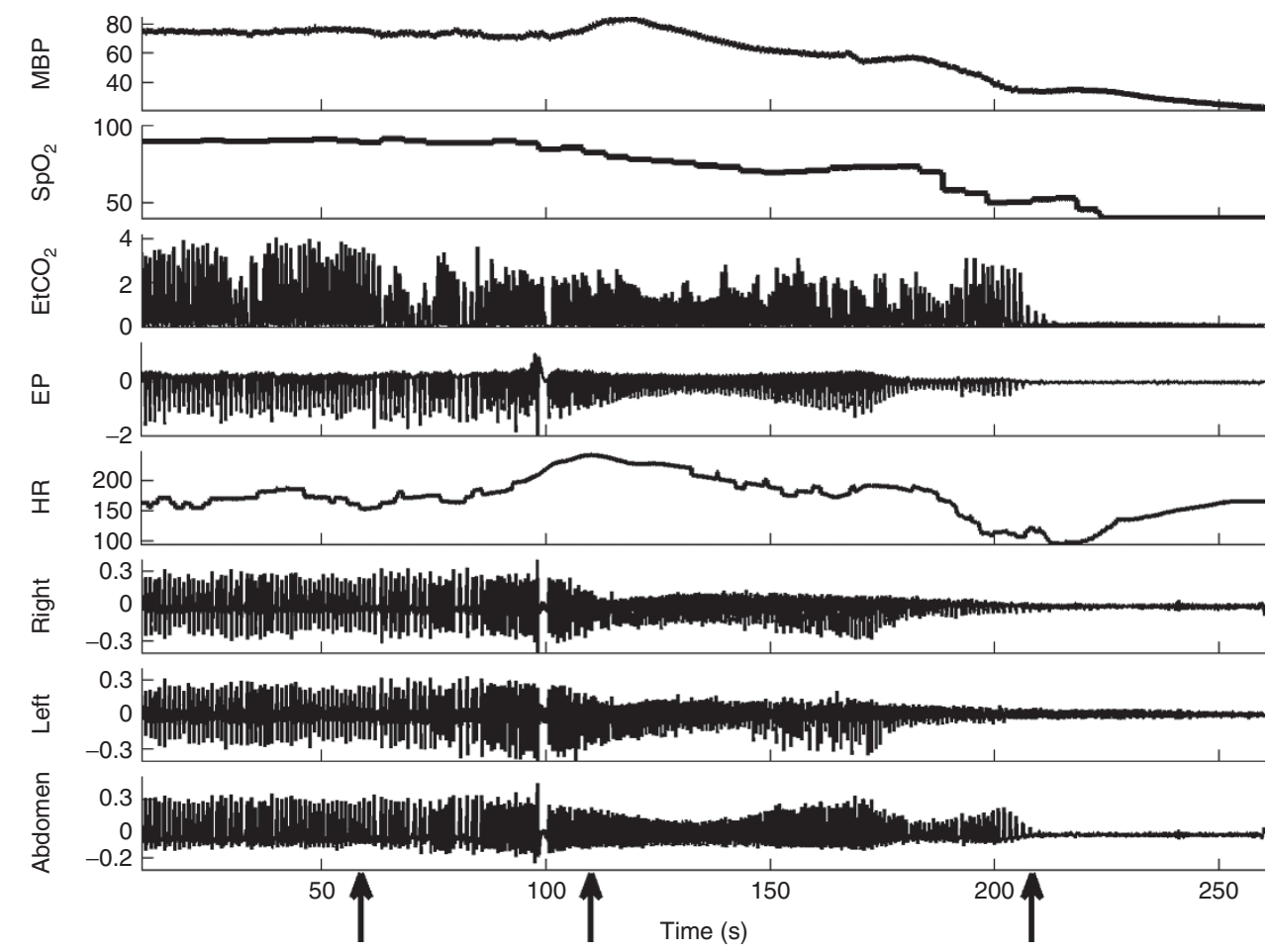

Figure 2. Injection of succinylcholine (first arrow) induced progressive hypopnea that developed to complete pseudo-CA (third arrow). The second arrow indicates the time of a $50 \%$ decrease in the esophageal pressure.



b

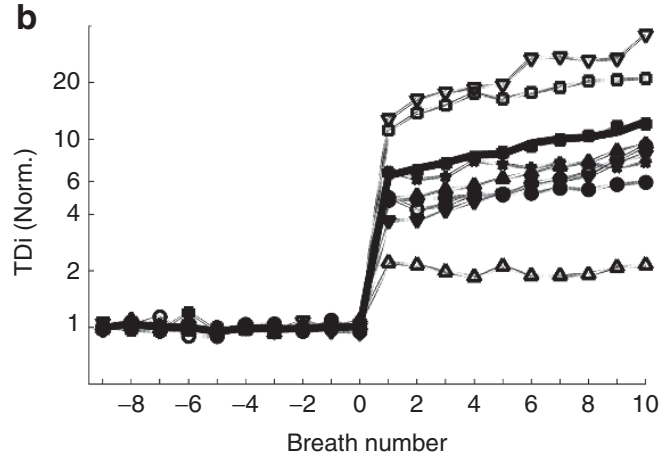

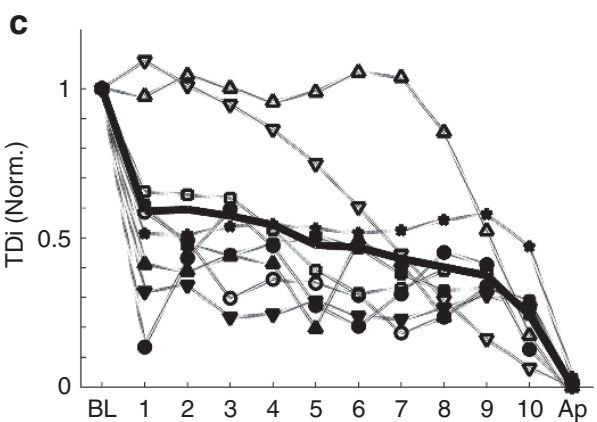

d

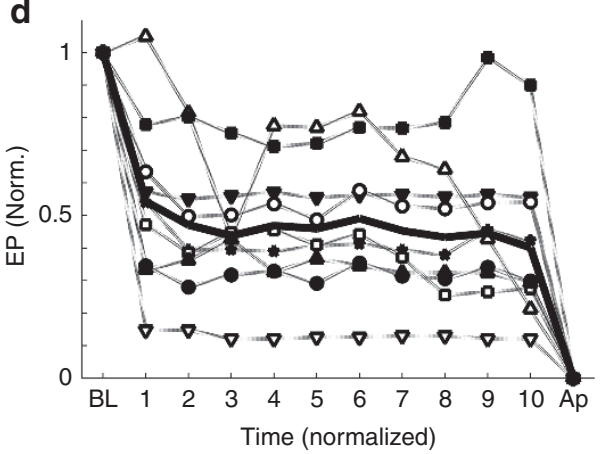

Figure 3. Similar changes in the tidal displacement index and the esophageal pressure, during both obstructive and central apneic events, were observed. Obstructive apnea generated an immediate increase in the (a) tidal displacement index and (b) the EP. Central hypopnea-apnea yielded a gradual decrease in (c) tidal displacement index and in the (d) EP. The values are normalized to mean baseline levels. Each rat is depicted by a different symbol.

\section{Breath Time Length}

$\mathrm{OA}$ induced a significant and consistent widening of the breath signals, as shown in Figure 4a. The breath time length (BTL) was $470 \pm 65 \mathrm{msec}$ at baseline and increased to $656 \pm 107 \mathrm{msec}$
$(+39.6 \pm 22.8 \% ; P<0.0002)$ after the obstruction. In the central hypopneic/apneic events, the BTL slightly increased from $436 \pm 78$ to $560 \pm 104 \mathrm{msec}$ after the injection of succinylcholine, as shown in Figure $4 \mathbf{b}$ but failed to reach any statistical significance $(P=0.83)$. 

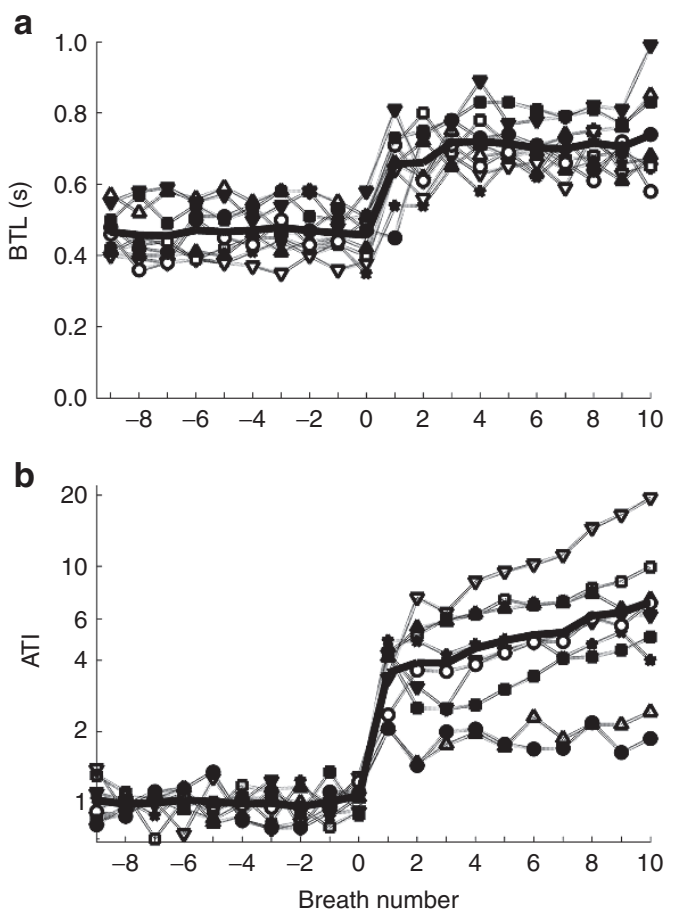

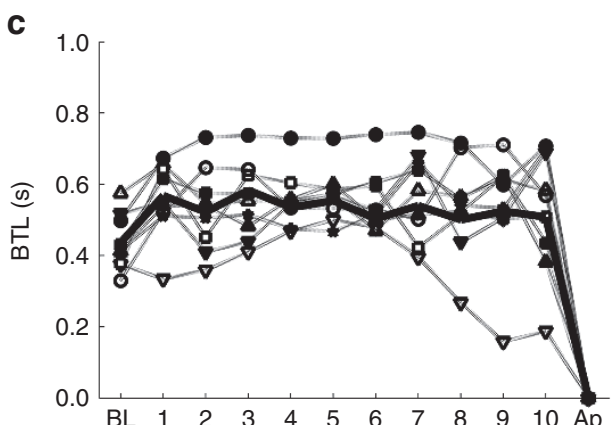

d

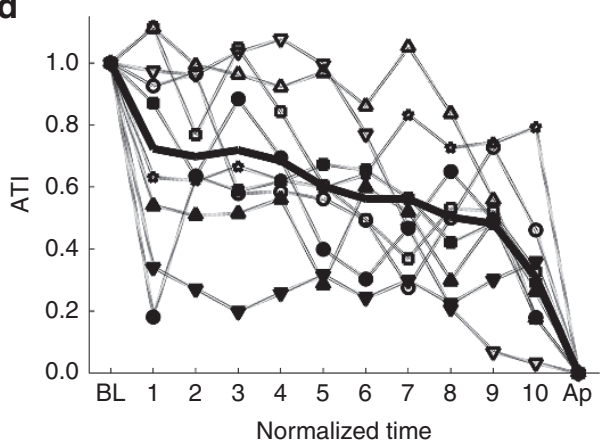

Figure 4. Obstructive and CAs have distinctive effects on the breath time length (BTL) and the amplitude time integral (ATI). Obstructive apnea induced an immediate increase in both $(\mathbf{a})$ the BTL $(P<0.0002)$ and $(\mathbf{b})$ the ATI. Central hypopnea-apnea yielded an insignificant increase in $(\mathbf{c})$ BTL $(P=0.83)$ and a gradual decrease in (d) the ATI.

\section{Amplitude Time Integral}

Figure $4 c, d$ depict the changes from baseline in the amplitude time integral (ATI) measured during the obstruction and hypopneic episodes, respectively. A mean $3.32 \pm 1.40-$ fold increase in ATI was observed upon airway obstruction (Figure 4c). The overt increase from baseline in the ATI was statistically significant $(P<0.024)$ in the first breath following obstruction, and even more so $(P<0.00001)$ in successive breaths. During the central hypopnea/apnea, the ATI progressively decreased to zero (Figure 4d). The mean correlation coefficient of ATI and EP was $0.956 \pm 0.036$ (range: 0.8860.989 ) for all obstructive episodes, and $0.957 \pm 0.039$ (range: 0.894-0.994) for all hypopneas during pseudo-CAs.

All the indices measured during the obstructive episodes are presented in Figure 5. Overt changes in EP and in the motion sensor indices were observed in all cases (100\% sensitivity, no false positive). Interestingly, a significant decrease in blood pressure (deviation $>20 \%$ ) was measured in all cases. This hemodynamic decompensation required relief of the obstruction even before a significant $(>5 \%)$ decline in the arterial $\mathrm{O}_{2}$ saturation $\left(\mathrm{SpO}_{2}\right)$ was detected, leading to a lower sensitivity (77\%) for the $\mathrm{SpO}_{2}$. The changes in the EP, $\mathrm{EtCO}_{2}$, and the motion sensor indices were detected immediately within the first breaths after initiation of obstruction $(1.41 \pm 0.30 \mathrm{~s}$ for EP and $1.07 \pm 0.26 \mathrm{~s}$ for TDi and ATI), as shown in Figure $5 \mathrm{~b}$. The hemodynamic changes (heart rate and $\mathrm{BP}$ ) and the decline in $\mathrm{SpO}_{2}$ were only observed more than $10 \mathrm{~s}$ after obstruction; a $5 \%$ decline in $\mathrm{SpO}_{2}$ was detected only after $13.3 \pm 5.2 \mathrm{~s}$. Thus, the changes in EP and
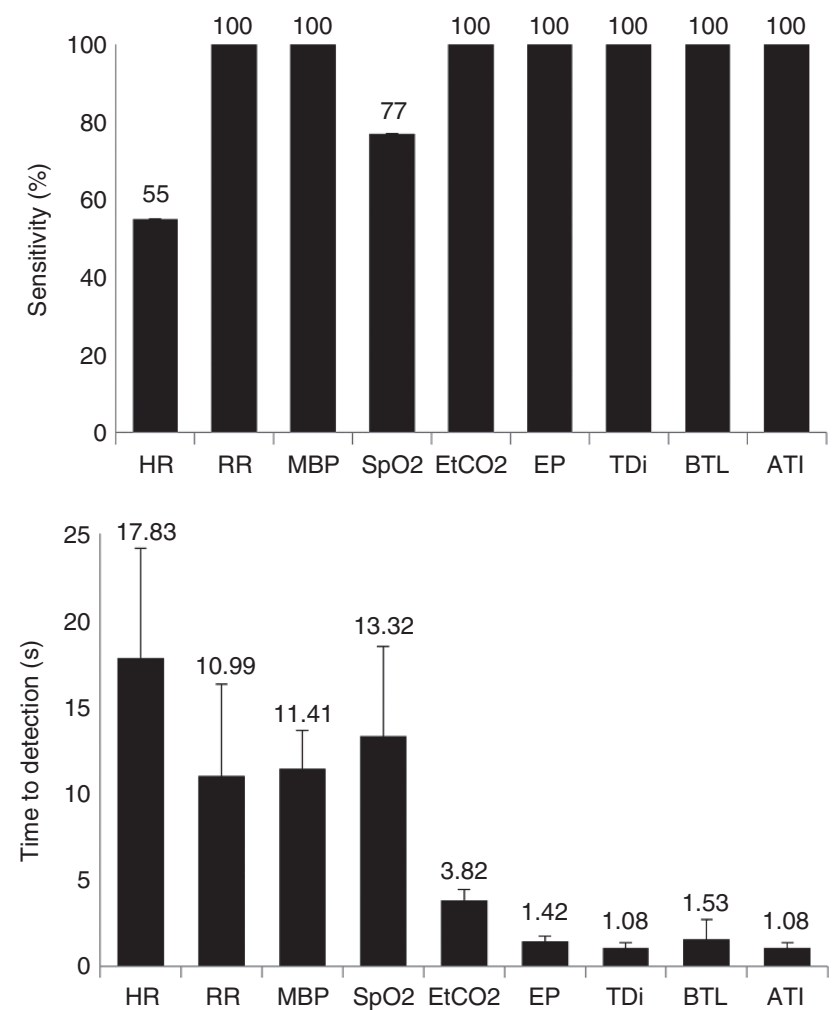

Figure 5. The EP and the motion sensor indices provide $100 \%$ (a) sensitivity and (b) rapid detection of the obstructive episode. ATI, amplitude time integral; $\mathrm{BTL}$, breath time length; $\mathrm{EP}$, esophageal pressure; $\mathrm{EtCO}_{2^{\prime}}$ end tidal $\mathrm{CO}_{2^{\prime}} ; \mathrm{HR}$, heart rate; $\mathrm{MBP}$, mean arterial pressure; $\mathrm{RR}$, respiratory rate; $\mathrm{SpO}_{2^{\prime}}$ pulse oximetry. 

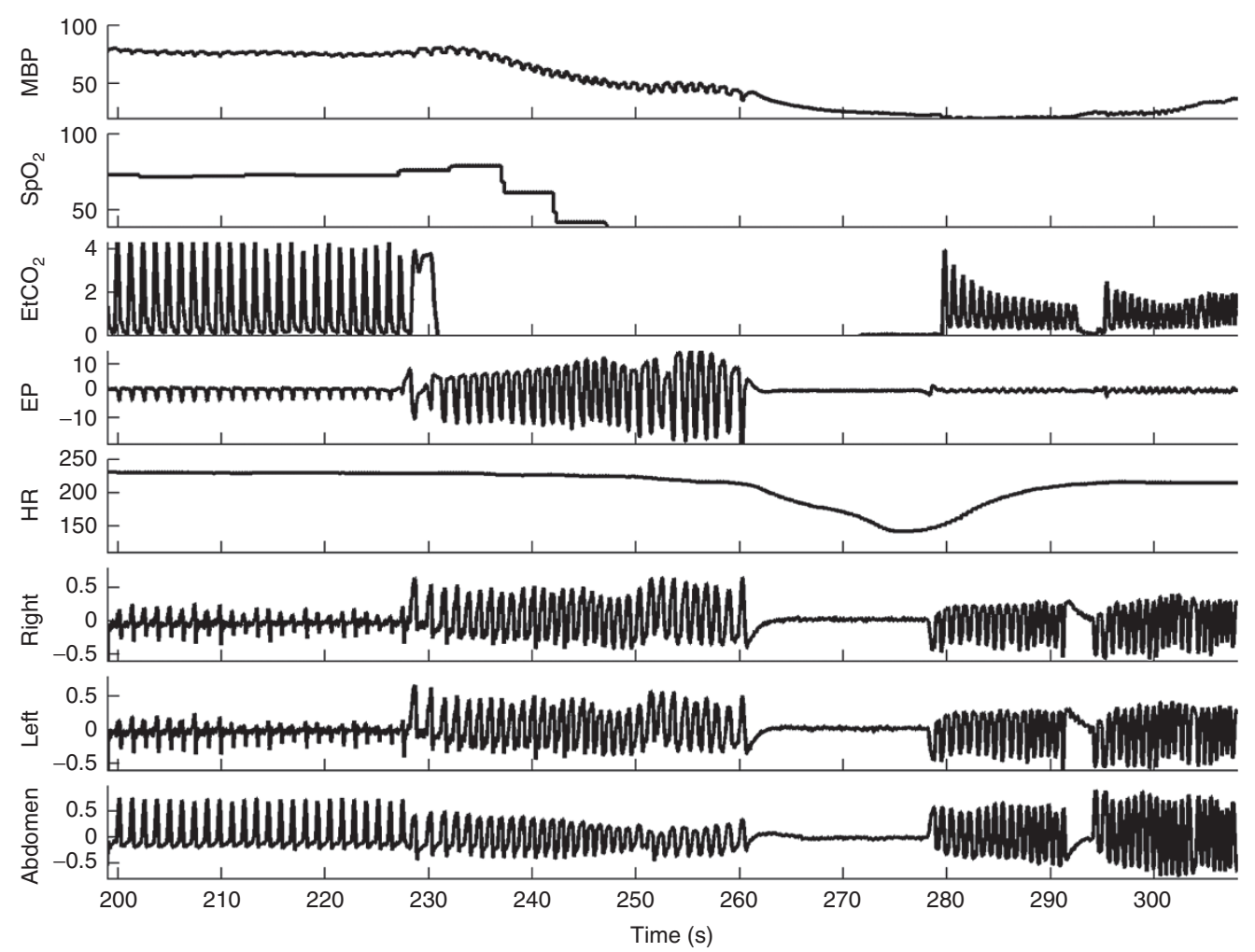

Figure 6. An episode of "near-fatal obstruction," where obstruction (from s 228 to 260) evolved into near-fatal central apneic episode (s 260 to 278 ). Note the abrupt transition from vigorous breaths to complete cessation of breath motion. The recorded movements seen from $t=278 \mathrm{~s}$ were from manual ventilation with a self-inflating bag.

in chest dynamics were not only the most sensitive but also enabled earlier detection of obstruction.

\section{Near-Fatal Obstruction Episodes}

Six of the $43 \mathrm{OA}$ events, in three of the nine rats, evolved into complete cessation of breathing efforts, as in CA. These events were associated with a severe decrease in $\mathrm{BP}$ and $\mathrm{SpO}_{2}$, as depicted in Figure 6. Although the obstruction was rapidly removed, spontaneous recovery did not occur. In all these cases, manual ventilation with a self-inflating bag was required to resuscitate the rats and to stop the vicious cycle, where OA yielded severe hypoxemia of the brain stem with CA. It is interesting to note that in all these six cases, there was an abrupt changeover from $\mathrm{OA}$ with vigorous chest movement, to $\mathrm{CA}$ with no noticeable respiratory motion.

\section{DISCUSSION}

The present study established the feasibility of rapid detection and classification of apneic episodes utilizing a novel noninvasive, respiration dynamics monitoring system that employs miniature motion sensors, in animals (rats) that are smaller than premature infants. The detection and classification of hypopnea, CA, and OA events were based on three simple and intuitive parameters: the TDi, BTL, and ATI. The measured changes in these parameters were in tight correlation with the changes in the esophageal pressure, during both obstructive and pseudocentral apneic episodes. These indices also enabled rapid detection of the obstructive episodes, within the first breath cycles, and thus can provide early alarm, before the development of severe respiratory failure (3-7).

Polysomnography, which measuring thoraco-abdominal dynamics in conjunction with airflow, is considered the gold standard for identifying and assessing the severity of apneic episodes $(4,10,16,20-22)$. While thoraco-abdominal asynchrony is expected during obstructive episodes in adults (25), neonates and infants may normally exhibit this type of breathing (10). In parallel to the reported modest success of polysomnography in detecting obstructive sleep apnea, some published works have demonstrated inaccurate detection in infants, with low specificity of $10.9 \%$ (ref. 10). Plethysmography is not in routine clinical practice in neonatal ICUs, since it is cumbersome in the ICU setting, and the bands cause friction and irritation of the tender infant skin, and may restrict the breathing of premature infants.

Monitoring chest wall dynamics using the mechanical indices presented here enables precise detection of the initiation and progression of OA s and identification of central hypopneic/apneic events in the rat model. We have shown in the past that TDi correlates with both tidal volume and inspiratory pressure $(23,24)$. Here, we show that TDi also correlates with the EP. Thus, the suggested TDi and ATI measurements can be used as a surrogate for the EP method, and can be obtained with small sensors suitable even for the smallest premature infants (24).

Airway obstruction induced a significant increase in the duration of each breath, as a consequence of the need to recruit 


\section{Articles | Waisman et al.}

and to increase the activity of the respiratory muscles in efforts to overcome the obstruction. OA in adults is associated with an increase in inspiratory time; a $10 \%$ prolongation of the inspiratory phase of the nasal flow, relative to baseline, can classify obstructive episodes with sensitivity and specificity of 72 and $77 \%$, respectively (26). In the present preclinical study, we obtained an overt $39.6 \pm 22.8 \%$ prolongation of the BTL that was extrapolated from chest wall dynamics measurements, and not from nasal airflow. Moreover, the method described here proved highly sensitive and successfully differentiated between obstructive and central apneic episodes. Obviously, the applicability of these preclinical findings and the suggested noninvasive monitoring method must be tested in clinical trials.

The integral of the amplitude of the chest wall movement over the breath cycle, denoted as the ATI, integrates the effects of the interventions on the amplitude and the length of the breath cycles. While the TDi provides information relating to the peak amplitude of the local displacement, the ATI assesses the respiratory energy expenditure. The ATI concept is founded on muscle physiology and is analogous to the "stress-time integral" that is used to assess skeletal or cardiac muscle energy expenditure $(27,28)$. During an isovolumic regime, cardiac energy consumption is proportional to the stress-time integral $(27,28)$. Similarly, in the presence of airway obstruction, respiratory muscles work at an apparently isovolumic regime.

CA can be easily detected by current monitoring systems that measure transthoracic impedance. However, hypopneic and obstructive apneic episodes are usually suspected only when bradycardia and hypoxemia are present, and the infants are already in severe distress or faint $(7,12-14,17,29)$. Repeated occurrences of hypoxemic episodes have been associated with impaired neurodevelopment, including mental retardation, cerebral palsy, blindness, growth retardation, and severe learning difficulties $(4,15,17,30,31)$. Thus, timely detection and characterization of hypopneic and apneic episodes is of utmost importance. Various modes of interventions have been explored in order to reduce hypoxemic episodes, including physical or sensory-neural stimulation (32), feeding practices (33), positioning $(15,17)$, appropriate pharmacological treatment (5), or respiratory support. However, one of the main obstacles lies in correct identification and quantification of the severity of the apneic episodes, in a simple and practical manner. A means of timely detection of hypopneic episodes, which evolve to hypoxemia and bradycardia in premature infants, is still lacking, while the incidence of such events is still very high (4). Many agree that with the current monitoring methods, it is impossible to unambiguously detect OA or distinguish between obstructive or CA without concomitantly measuring airflow or nasal end-tidal $\mathrm{CO}_{2}$ and respiratory effort $(10,34)$. Our results strongly suggest that a distinction between obstructive and central apneic episodes can be achieved by monitoring the trends in TDi, BTL and ATI.

During our experiments, we documented episodes of "near-fatal obstruction", where short obstructive apneic episodes evolved into a terminal event of CA. An interesting phenomenon was observed; the transition from OA, with vigorous breaths, to complete CA was abrupt and unpredicted. There was no sign of fatigue, slowed breath rate or gradual decrease in the breath amplitude. In all six events of "near-fatal apnea," the transitions to complete CA were instantaneous.

Pure obstructive events account for $4-13 \%$ of all apneic episodes, while $40-50 \%$ are mixed apneic episodes, in which a CA precedes OA $(1-3,29)$. The "near-fatal obstruction" observed here has not been described in the literature, and differs from the mixed apnea since the CA followed the OA. Moreover, in mixed apnea, the CA initiates from normal saturation, while in the "near-fatal obstruction," the CA evolved when there was already severe hypoxemia secondary to the $\mathrm{OA}$, and is likely due to hypoxia in the medullary respiratory center. The significant advantages provided by the suggested monitoring modality include rapid detection and classification of the apneic episodes, within a few breath cycles, before the onset of severe hypoxemia and bradycardia. Obviously, these "near-fatal obstruction" events require more thoroughly investigations which fall beyond the scope of the current presentation.

\section{Limitations}

This study was performed in healthy rats, weighing $250-300 \mathrm{~g}$, and therefore the translation to premature infants and to adults is not straightforward. However, the study aimed to investigate the utility of a noninvasive and simple means of early detection and classification of all types of apneic episodes. Apneic episodes are frequent in neonatal rat pups and other animal models, but are most probably of central type (depressed muscle activation) due to late development of the central and peripheral chemoreceptors $(2,35)$. Therefore, we imposed unequivocal obstructive and central-type episodes in adult rats to test the feasibility of real-time detection and classification of these two distinct perturbations.

CA was induced by administration of a peripheral depolarizing muscle relaxant. Although this model was used by others $(36,37)$, the pharmacokinetics of succinylcholine and its effects on the different respiratory muscles are obviously different from the dynamics of the respiratory muscles activation by the medullary respiratory center and the neural respiratory control system.

\section{Conclusion}

Continuous monitoring of chest wall dynamics, with noninvasive and miniature sensors, enables quantification of ventilation dynamics and immediate detection and characterization of episodes of OA, as well as hypopnea and CA. Monitoring the suggested novel mechanical indices enables quantification of the respiratory effort which demonstrated congruence with the cumbersome and moderately invasive measurement of the esophageal pressure. This study calls for further investigation of the presented method in clinical studies, to verify it applicability as a noninvasive alternative to esophageal pressure measurements, for detection and classification of apneic episodes and assessment of their severity. 


\section{METHODS}

This study was approved by the Technion-Israel Institute of Technology Ethics Committee for the Care and Use of Animals. Male Sprague-Dawley rats were anesthetized using an intraperitoneal injection of ketamine $90 \mathrm{mg} / \mathrm{kg}$, xylazine $10 \mathrm{mg} / \mathrm{kg}$, and acepromazine (ACP) $10 \mathrm{mg} / \mathrm{kg}$. A venous line was placed in the tail vein and a femoral arterial line was placed for blood pressure monitoring and gas sampling (Roche OPTI CCA, Mannheim, Germany). A tracheostomy tube was inserted and connected to a side stream end-tidal $\mathrm{CO}_{2}\left(\mathrm{EtCO}_{2}\right)$ monitor (Datex MultiCup, Helsinki, Finland). Rectal temperature was monitored and maintained at $36.0-37.5^{\circ} \mathrm{C}$, using a heating pad. Esophageal pressure (EP) was measured using a fluidfilled catheter (PE-50 polyethylene tubing) positioned at the midesophagus and connected to a pressure sensor (Millar Instruments, Houston, TX).

As previously described $(23,24)$, chest wall and abdominal displacement were continuously monitored using three miniature accelerometers (Pneumedicare, Yokneam, Israel) that were placed on the right and left sides of the chest (midclavicular line at the forth intercostal space) and epigastrium. These accelerometers enable measurement of the local subsonic (below $20 \mathrm{~Hz}$ ) wall motion, as well as the vibrations from the breath and heart sounds. From these signals, movement amplitude and breath time length can be measured. ECG, blood pressure (BP), EtCO, and $\mathrm{SpO}_{2}$ were continuously acquired and displayed on an anesthesia/ ICU monitor (Type CU8; Datex Ohmeda, Madison, WI).

\section{Experimental Protocol}

$\mathrm{OA}$ and CA episodes were induced, as detailed below, in each rat, and after each intervention, recordings continued until complete recovery to baseline values of all vital signs (heart rate, $\mathrm{BP}, \mathrm{EtCO}_{2}$, and $\mathrm{SpO}_{2}$ ).

\section{Obstructive Apnea}

OA was achieved by complete airway obstruction with an occluded endotracheal tube connector. Each episode of OA lasted no more than 30 seconds or until an overt decrease in $\mathrm{BP}$ or $\mathrm{SpO}_{2}$ occurred. Time zero was defined as the time of endotracheal tube occlusion.

\section{Central Hypopnea and CA}

CA was achieved by intravenous infusion of $0.1 \mathrm{mg} / \mathrm{kg}$ succinylcholine (Succamethonium, Labessal, Portugal), as reported by others $(36,37)$. Succinylcholine-induced progressive muscular paralysis evolved into transient complete apnea. If apnea did not occur after the initial infusion, the same dose was administered again. Hypopnea was defined as a decrease of $>50 \%$ in the respiratory effort, as determined by the EP, relative to baseline (38).

Time zero for the succinylcholine-induced CA episode was defined as the time when the first intravenous succinylcholine infusion was initiated. Because the response to succinylcholine significantly varied between animals, the time axis corresponding to hypopnea/CA was normalized and divided into 10 consecutive intervals of equal duration. The end of the tenth time segment was defined as the time point at which no respiratory motions were observed (CA). The mean amplitude of each index was calculated for each time segment of the succinylcholine-induced episodes.

The following indices were used for data analysis:

TDi: the TDi represents the amplitude of the local tidal displacement during the breath cycle, at the site of measurement (right or left side of the chest and epigastric area).

BTL: the BTL is the total duration of the inspiratory and the rapid expiratory phases in each breath cycle signal.

ATI: the ATI is the integral of the instantaneous tidal displacement over time, along the entire respiratory cycle.

\section{Statistical Analysis}

Results are presented as mean \pm SD. Differences within groups were assessed using the paired Student's $t$-test. A $P$ value smaller than 0.05 was considered significant. A change greater than $20 \%$ from baseline was considered a significant deviation for the mean blood pressure, heart rate, respiratory rate, and end tidal $\mathrm{CO}_{2}$. Significant deviation for the $\mathrm{SpO}_{2}$ was defined as a decline greater than $5 \%$ from the baseline level. As for the motion sensor parameters (TDi, ATI, BTL), an increase greater than $20 \%$ from baseline was used for determination of obstructive episodes, and a decrease greater than 50\% defined central hypopneic/apneic episodes. The correlation coefficients between TDi and EP, and between ATI and EP, for both OAs and central hypopneas, were calculated for all experiments. The sensitivity is defined as the ratio of the true positive detections to the total imposed events, based on the above defined threshold levels.

\section{SUPPLEMENTARY MATERIAL}

Supplementary material is linked to the online version of the paper at http://www.nature.com/pr

\section{STATEMENT OF FINANCIAL SUPPORT}

This work was supported in part by a grant for the promotion of research at the Technion, Israel Institute of Technology, Haifa, Israel (A.L.). Chest wall dynamics were measured by equipment provided by Pneumedicare Ltd., Yokneam, Israel.

Disclosure: D.W., C.L., A.F., I.C.K., and A.L. are involved in the development of a respiratory function monitor within a collaborative effort between the Technion-Israel Institute of Technology, Clalit Health Services and Pneumedicare Ltd. L.L., H.B., and A.R. do not have conflict of interest to declare.

\section{REFERENCES}

1. Abu-Shaweesh JM. Respiratory disorders in preterm and term infants. In: Martin RJ, Fanaroff AA, Walsh MC, eds. Fanaroff and Martin's NeonatalPerinatal Medicine: Diseases of the Fetus and Infant. 9th edn. Elsevier, Philadelphia, PA, 2011:1141-50.

2. Abu-Shaweesh JM, Martin RJ. Neonatal apnea: what's new? Pediatr Pulmonol 2008;43:937-44.

3. Miller MJ, Martin RJ. Pathophysiology of apnea of prematurity. In: Polin R, Fox W, and Abman S eds. Fetal and Neonatal Physiology, 4th edn. Philadelphia, PA: Saunders-Elsevier, 2011:998-1011.

4. Martin RJ, Wang K, Köroğlu O, Di Fiore J, Kc P. Intermittent hypoxic episodes in preterm infants: do they matter? Neonatology 2011;100:303-10.

5. Schmidt B, Anderson PJ, Doyle LW, et al.; Caffeine for Apnea of Prematurity (CAP) Trial Investigators. Survival without disability to age 5 years after neonatal caffeine therapy for apnea of prematurity. JAMA 2012;307:275-82.

6. Dewolfe CC. Apparent life-threatening event: a review. Pediatr Clin North Am 2005;52:1127-46, ix.

7. Fu LY, Moon RY. Apparent life-threatening events (ALTEs) and the role of home monitors. Pediatr Rev 2007;28:203-8.

8. Shapiro-Mendoza CK, Kimball M, Tomashek KM, Anderson RN, Blanding S. US infant mortality trends attributable to accidental suffocation and strangulation in bed from 1984 through 2004: are rates increasing? Pediatrics 2009;123:533-9.

9. Moon RY. Technical report. SIDS and other sleep-related infant deaths: expansion of recommendations for a safe infant sleeping environment. Pediatrics 2011;128:e1341-67.

10. De Groote A, Groswasser J, Bersini H, Mathys P, Kahn A. Detection of obstructive apnea events in sleeping infants from thoracoabdominal movements. J Sleep Res 2002;11:161-8.

11. Sharma PB, Baroody F, Gozal D, Lester LA. Obstructive sleep apnea in the formerly preterm infant: an overlooked diagnosis. Front Neurol 2011;2:73.

12. Poets CF, Meny RG, Chobanian MR, Bonofiglo RE. Gasping and other cardiorespiratory patterns during sudden infant deaths. Pediatr Res 1999;45:350-4.

13. Poets CF. Apparent life-threatening events and sudden infant death on a monitor. Paediatr Respir Rev 2004;5:Suppl A:S383-6.

14. Harper RM. Sudden infant death syndrome: a failure of compensatory cerebellar mechanisms? Pediatr Res 2000;48:140-2.

15. Katz ES, Mitchell RB, D’Ambrosio CM. Obstructive sleep apnea in infants. Am J Respir Crit Care Med 2012;185:805-16.

16. Weese-Mayer DE, Corwin MJ, Peucker MR, et al. Comparison of apnea identified by respiratory inductance plethysmography with that detected by end-tidal CO(2) or thermistor. The CHIME Study Group. Am J Respir Crit Care Med 2000;162(2 Pt 1):471-80.

17. Zhao J, Gonzalez F, Mu D. Apnea of prematurity: from cause to treatment. Eur J Pediatr 2011;170:1097-105. 


\section{Articles Waisman et al.}

18. Argod J, Pépin JL, Smith RP, Lévy P. Comparison of esophageal pressure with pulse transit time as a measure of respiratory effort for scoring obstructive nonapneic respiratory events. Am J Respir Crit Care Med 2000;162:87-93.

19. Kushida CA, Giacomini A, Lee MK, Guilleminault C, Dement WC. Technical protocol for the use of esophageal manometry in the diagnosis of sleep-related breathing disorders. Sleep Med 2002;3:163-73.

20. Flemons WW, Littner MR, Rowley JA, et al. Home diagnosis of sleep apnea: a systematic review of the literature. An evidence review cosponsored by the American Academy of Sleep Medicine, the American College of Chest Physicians, and the American Thoracic Society. Chest 2003;124:1543-79.

21. Kahlke PE, Witmans MB, Alabdoulsalam T, Young R, MacLean JE, Mandhane PJ. Full-night versus $4 \mathrm{~h}$ evening polysomnography in children less than 2years of age. Sleep Med 2013;14:177-82.

22. Kirk VG, Bohn SG, Flemons WW, Remmers JE. Comparison of home oximetry monitoring with laboratory polysomnography in children. Chest 2003;124:1702-8.

23. Waisman D, Faingersh A, Levy C, et al. Early detection of deteriorating ventilation by monitoring bilateral chest wall dynamics in the rabbit. Intensive Care Med 2012;38:120-7.

24. Waisman D, Levy C, Faingersh A, et al. A new method for continuous monitoring of chest wall movement to characterize hypoxemic episodes during HFOV. Intensive Care Med 2011;37:1174-81.

25. Al-Angari HM, Sahakian AV. Automated recognition of obstructive sleep apnea syndrome using support vector machine classifier. IEEE Trans Inf Technol Biomed 2012;16:463-8.

26. Mooney AM, Abounasr KK, Rapoport DM, Ayappa I. Relative prolongation of inspiratory time predicts high versus low resistance categorization of hypopneas. J Clin Sleep Med 2012;8:177-85.

27. Han JC, Taberner AJ, Tran K, et al. Relating components of pressurevolume area in Suga's formulation of cardiac energetics to components of the stress-time integral. J Appl Physiol (1985) 2012;113:988-95.
28. Sela G, Landesberg A. The external work-pressure time integral relationships and the afterload dependence of Frank-Starling mechanism. J Mol Cell Cardiol 2009;47:544-51.

29. Fajardo C, Alvarez J, Wong A, Kwiatkowski K, Rigatto H. The incidence of obstructive apneas in preterm infants with and without bronchopulmonary dysplasia. Early Hum Dev 1993;32:197-206.

30. Janvier A, Khairy M, Kokkotis A, Cormier C, Messmer D, Barrington KJ. Apnea is associated with neurodevelopmental impairment in very low birth weight infants. J Perinatol 2004;24:763-8.

31. Pillekamp F, Hermann C, Keller T, von Gontard A, Kribs A, Roth B. Factors influencing apnea and bradycardia of prematurity - implications for neurodevelopment. Neonatology 2007;91:155-61.

32. Bloch-Salisbury E, Indic P, Bednarek F, Paydarfar D. Stabilizing immature breathing patterns of preterm infants using stochastic mechanosensory stimulation. J Appl Physiol (1985) 2009;107:1017-27.

33. Corvaglia L, Spizzichino M, Aceti A, et al. A thickened formula does not reduce apneas related to gastroesophageal reflux in preterm infants. Neonatology 2013;103:98-102.

34. Hammer J, Newth CJ. Assessment of thoraco-abdominal asynchrony. Paediatr Respir Rev 2009;10:75-80.

35. Peng YJ, Rennison J, Prabhakar NR. Intermittent hypoxia augments carotid body and ventilatory response to hypoxia in neonatal rat pups. J Appl Physiol (1985) 2004;97:2020-5.

36. Chen L, Scharf SM. Comparative hemodynamic effects of periodic obstructive and simulated central apneas in sedated pigs. J Appl Physiol (1985) 1997;83:485-94.

37. Tarasiuk A, Scharf SM. Cardiovascular effects of periodic obstructive and central apneas in dogs. Am J Respir Crit Care Med 1994;150:83-9.

38. Luo YM, Tang J, Jolley C, et al. Distinguishing obstructive from central sleep apnea events: diaphragm electromyogram and esophageal pressure compared. Chest 2009;135:1133-41. 